\title{
Realidad nacional de los programas de detección auditiva temprana con miras a la cobertura universal
}

\author{
Nationwide status of early hearing detection programs: \\ Aiming for universal coverage
}

\author{
Cecilia Sedano $\mathbf{M}^{1}$, Alex San Martín U², Maritza Rahal E
}

\begin{abstract}
RESUMEN
Introducción: El 50\% de los recién nacidos hipoacúsicos no presenta ningún factor de riesgo, por lo que la pesquisa universal y temprana puede cambiar el pronóstico de estos pacientes. La detección auditiva precoz universal es la realización de un examen para descartar hipoacusia a todos los recién nacidos vivos de un centro determinado.

objetivo: Describir y analizar la presencia de programas de pesquisa auditiva universal en Chile y las características de los programas implementados.

Material y método: Se identificó a los centros con programas de detección auditiva universal en Chile y se les envió una encuesta electrónica acerca de la estructuración de cada programa.

Resultados: 34 hospitales cuentan con programas establecidos; 31 contestaron la encuesta; $73 \%$ inició el programa después de 2010; 46\% se encuentra financiado por el Ministerio de Salud; la gran mayoría está a cargo de fonoaudiólogos y tecnólogos médicos. Los principales métodos de detección disponibles son emisiones otoacústicas por producto de distorsión y potenciales evocados auditivos de tronco cerebral automatizado. En cuanto al diagnóstico definitivo el $71 \%$ realiza con potenciales evocados auditivos de tronco cerebral estímulo click más impedanciometría.

Conclusión: Los hospitales con detección auditiva precoz universal corresponden al $50 \%$ de los hospitales con maternidad y se encuentran principalmente en la zona central. A pesar de que existe interés y avance por parte de algunos centros hospitalarios en responder a la necesidad de estos programas, aún queda mucho por hacer, siendo el objetivo principal una cobertura universal a nivel nacional.
\end{abstract}

Palabras clave: Congénito, hipoacusia, recién nacido, detección auditiva precoz, salud pública.

\section{ABSTRACT}

Introduction: $50 \%$ of hearing-impared newborns do not have any associated risk factors; so early universal detection programs can change the prognosis of these pa-

\footnotetext{
1 Médico del Servicio de Otorrinolaringología Hospital Barros Luco Trudeau, Santiago, Chile. Universidad de Chile, Campus Sur.

2 Fonoaudiólogo Servicio Otorrinolaringología Hospital Barros Luco Trudeau, Santiago, Chile.
}

Recibido el 10 de julio de 2017. Aceptado el 2 de septiembre de 2017. 
tients. Universal newborn hearing screening consists in studying all newborns in a particular area, regardless of the presence of risk factors.

Aim: Describe and analyze the presence of universal newborn hearing screening programs in Chile and its characteristics.

Material and method: We identify establishments that have universal newborn hearing screening programs and sent them An electronic survey focusing on the structure of each program.

Results: 34 hospitals have universal newborn hearing screening. Of these, 31 answered the survey.73\% begun the program after 2010. The Ministry of Health provides financing in $46 \%$. Audiologists and medical technicians majoring in otolariyngology are in charge of the vast majority of the programs. Available screening methods are distortion product otoacoustic emissions (90\%) and automated Brainsteam evoked response audiometry (67.8\%). As for the definitive diagnosis, $71 \%$ use Brainsteam evoked response audiometry click plus impedanciometry.

Conclusion: $50 \%$ of the hospitals with maternities have universal hearing screening; most of them are in Chile's central area. Even though some hospitals show interest and have made advances in this regard, there is still much to be done, being universal coverage the main objective.

Key words: Congenital, Hearing loss, Newborn, Early hearing screening, Public health.

\section{INTRODUCCION}

La hipoacusia sensorioneural congénita se encuentra en 1 a 3 por cada 1.000 recién nacidos vivos (RNV) ${ }^{1}$, llegando a 8 por 1.000 en pacientes que pasaron por la Unidad de Cuidados Intensivos Neonatal 0 con factores de riesgo ${ }^{2}$. Es 200 veces más frecuente que la fenilcetonuria $(1: 21.000)$ y 3,5 veces más que el hipotiroidismo congénito (1:3.500), patologías que cuentan con programas de pesquisa universal en nuestro país ${ }^{3}$.

Actualmente en Chile dentro de las patologías cubiertas por las Garantías Explícitas en Salud (GES) se encuentra la hipoacusia sensorioneural bilateral del prematuro, que incluye un programa de detección auditiva temprana a todos los RNV de menos de 32 semanas de gestación o que pesen menos de 1.500 gramos al nacer 4 .

Dentro de los factores de riesgo conocidos para hipoacusia congénita se encuentran la prematurez extrema, bajo peso de nacimiento, infecciones congénitas, uso de drogas en el embarazo, entre otras ${ }^{5}$. Sin embargo, se ha visto que el $50 \%$ de los niños con hipoacusia no presentan factores de riesgo identificables, por lo que estarían fuera de este programa de pesquisa auditiva ${ }^{6}$.

Desde el año 1994 el organismo americano encargado de las recomendaciones de identifica- ción temprana de hipoacusia, Joint Committee on Infant Hearing $(\mathrm{JCIH})$, se ha referido al programa de pesquisa precoz 0 tamizado auditivo universal (TAU) neonatal 7 .

EI TAU consiste en realizar un examen de detección de hipoacusia a todos los recién nacidos vivos de un centro o zona determinada, independiente de los factores de riesgo. El objetivo según el JCIH es identificar niños con hipoacusia, intervenir precozmente y maximizar las competencias lingüísticas, alfabetismo y desarrollo psicosocial?

Se considera un programa eficiente cuando tiene una cobertura mayor al $95 \%$, el porcentaje de referidos a diagnóstico es menor al $4 \%$ y que el tratamiento sea cercano al $100 \%$.

En estos programas se realiza un examen de pesquisa inicial a todos los RNV con emisiones otoacústicas (EOA) evocadas o con PEAT (potenciales evocados auditivos de tronco cerebral) de detección automatizado con estímulo click dependiendo el caso. Los pacientes que no pasan este examen tienen sospecha de hipoacusia, por lo que se repite una vez más, quienes nuevamente no pasan son derivados para realizar un examen diagnóstico, que se puede realizar con PEAT diagnóstico con estímulo click, tone burst, chirp, pip; PEAT óseo, potenciales de estado estable. 
Los pacientes diagnosticados con hipoacusia deben iniciar el tratamiento y seguimiento correspondiente.

En Chile algunos centros tanto públicos como privados, motivados por diversas causas, han iniciado la implementación de detección universal en forma autónoma8.

\section{OBJETIVO}

El objetivo del presente estudio es describir y analizar la implementación de TAU a nivel nacional y las características de los programas existentes a la fecha.

\section{MATERIAL Y MÉTODO}

Durante el primer y segundo trimestre del año 2016 se contactó telefónicamente a todos los hospitales públicos y de Fuerzas Armadas de Chile con maternidad, para preguntar si realizaban TAU y se identificó al o los encargados del programa de cada centro y se les envió una encuesta electrónica de 9 preguntas acerca de la estructuración de éste.
Se excluyeron los centros privados por no realizar TAU propiamente tal, ya que en aquellos centros la pesquisa se considera como un servicio electivo que debe ser costeado por el paciente.

Se realizó un análisis descriptivo de los datos.

\section{RESULTADOS}

De los 219 hospitales en Chile, se identificaron 68 centros que contaban con maternidad y fueron contactados. De éstos, 34 hospitales refirieron realizar TAU (13 de 15 regiones del país), a los cuales se les envió la encuesta electrónica. Del total de centros, 31 recintos hospitalarios contestaron la encuesta y 3 declinaron participar del estudio (Tabla 1).

En la encuesta realizada, se preguntó acerca del año de inicio de los programas, financiamiento, entre otros.

Se encontró que es una iniciativa relativamente nueva, ya que el 83,8\% comenzó el programa posterior al año 2010. El recinto más precoz, inició el programa de detección auditiva universal en el año 2000.

En cuanto al financiamiento para el programa, en el $55 \%$ de los casos los recursos provienen de

Tabla 1. Resultados

\begin{tabular}{|ll|}
\hline Hospitales con TAU & 34 (31 responden encuesta) \\
RNV con TAU & 73.000 apróx. \\
Financiamiento & $17(54.8 \%)$ \\
Hospital & $6(19,35 \%)$ \\
Universidad & $5(16 \%)$ \\
Chile crece contigo & $3(9,6 \%)$ \\
Institución (Fuerzas Armadas, Carabineros) & $29(93,5 \%)$ \\
Examen de pesquisa & $7(22,5 \%)$ \\
EOA producto de distorsión & $20(64,5 \%)$ \\
EOA transientes & $11(35,5 \%)$ \\
PEAT automatizados con estímulo click & $18(58 \%)$ \\
Profesional pesquisa & $2(6,5 \%)$ \\
Tecnólogo médico mención otorrinolaringología & $24(77,4 \%)$ \\
Fonoaudiólogo & $13(41,9 \%)$ \\
Matrona & \\
Examen diagnóstico & $4(12,9 \%)$ \\
PEAT con estímulo click más impedanciometría & $7(19,3 \%)$ \\
PEAT con estímulo tone burst, chirp o pip & \\
Otros (PEAT óseo, impedanciometría de & 15 \\
alta frecuencia, potenciales de estado estable) & 11 \\
Derivación a otro centro & \\
Profesional diagnóstico & \\
Tecnólogo médico mención otorrinolaringología & \\
Fonoaudiólogo &
\end{tabular}


Ios propios hospitales (17), 19\% de universidades (6), $16 \%$ del programa Chile Crece Contigo (5). El $10 \%$ restante corresponde a instituciones de las Fuerzas Armadas y Carabineros.

En nuestro estudio encontramos que se está realizando TAU a un promedio aproximado de 73.000 RNV por año, según datos del Ministerio de Salud el número de RNV anual en hospitales públicos es de $120.000^{9}$.

Dentro de los exámenes con los que contaba cada centro para realizar la detección auditiva universal, se vio que las EOA producto de distorsión están en el 93,5\% (29) de los centros, las E0A transientes en el $22,5 \%$ (7) y los PEAT automatizados con estímulo click en $64,5 \%$ (20) de los centros.

Se preguntó sobre la presencia de personal administrativo encargado del programa, solo 6 centros refirieron tener secretaria dedicada al proyecto.

Respecto del profesional que realiza el examen inicial de detección auditiva, en 58\% de los casos fue realizado por fonoaudiólogos, $36 \%$ por tecnólogos médicos mención otorrinolaringología y solo $6 \%$ por matronas.

A diferencia del estudio inicial, el profesional que realiza el examen diagnóstico en niños que ya fueron referidos, el $60 \%$ corresponde a tecnólogos médicos mención otorrinolaringología y el $40 \%$ fonoaudiólogos.

Para el examen diagnóstico, 77,4\% (24) de los centros cuentan con PEAT con estímulo click asociado a impedanciometría; el 41,9\% (13) con PEAT con estímulo tone burst, chirp o pip. El 19,3\% (6) de los centros derivan a la red correspondiente y $12,9 \%$ cuentan con otros exámenes adicionales como PEAT óseo, impedanciometría de alta frecuencia y potenciales de estado estable.

\section{DISCUSION}

Al igual como lo muestra la literatura internacional, en Chile se han descrito tasas de incidencia de hipoacusia de 3-4 casos cada 1.000 RNV $^{10}$. Si consideramos que el número de partos anuales en Chile es alrededor de 250.000 , incluyendo tanto instituciones públicas como privadas ${ }^{11}$, tenemos un grupo de niños no despreciable eventualmente con esta patología.
De los niños diagnosticados, como mencionamos previamente, el $50 \%$ no presenta factores de riesgo $0^{6}$, por lo que el TAU sería una herramienta de gran ayuda. La implementación de estos progra-

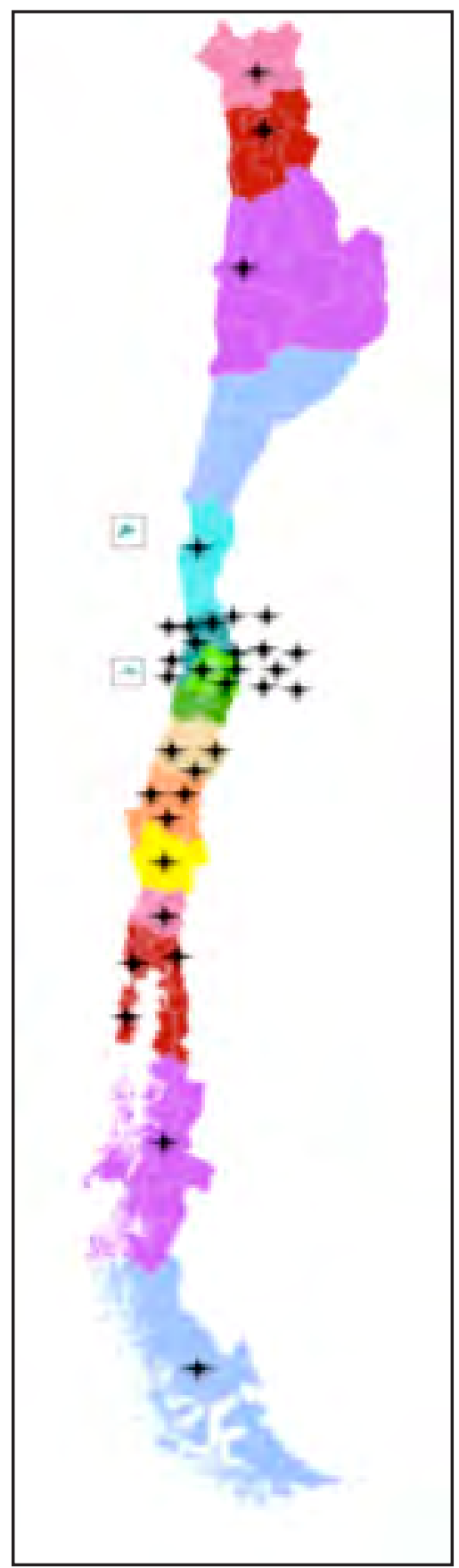

Figura 1. Distribución de centros que realizan TAU en Chile. 
mas permite un diagnóstico e inicio de terapia a edades significativamente más tempranas; lo que se reflejará en mejores habilidades comunicacionales y de lectoescritura?

Hay publicaciones que describen que la adición de una secretaria dedicada únicamente al proyecto de detección auditiva universal y transporte gratis al centro de estudio, aumentaría el seguimiento en $10 \%$ a $15 \%{ }^{12,13}$. En nuestra experiencia como hospital creemos que el seguimiento también ha mejorado desde que contamos con secretaria para el programa.

\section{CONCLUSION}

Considerando que los programas de TAU no son una medida obligatoria en nuestro país, los datos

\section{BIBLIOGRAFIA}

1. Grill E, Hessel F, Siebert U, Schnell-Inderst P, Kunze S, Nickisch A et al. Comparing the clinical effectiveness of different newborn hearing screening strategies. A decision analysis. BMC Public Health 2005; 31: 5-12.

2. Year 2007 Position Statement: Principles and Guidelines for Early Hearing Detection and Intervention Programs Joint Committee on Infant Hearing Pediatrics 2007; 120(4): 898921.

3. Dalzell L, Orlando M, MacDonald M, et al. The New York State universal newborn hearing screening demonstration project: ages of hearing loss identification, hearing aid fitting, and enrollment in early intervention 2000; 21: 118-30.

4. Ministerio de Salud. Guía Clínica Hipoacusia Neurosensorial Bilateral del Prematuro Santiago: Minsal, 2009.

5. American Academy of Pediatrics, Joint Committee on Infant Hearing. Year 2007 position statement: principles and guidelines for early hearing detection and intervention programs. Pediatrics 2007; 120: 898-921. recopilados en este estudio reflejan un avance respecto al reconocimiento de la hipoacusia congénita como una patología relevante, ya que se cuenta con programas implementados en 13 de las 15 regiones del país. Sin embargo, la gran mayoría están distribuidas en la zona central del Chile (Figura 1), quedando con menor cobertura el norte y sur del país. Además, solo el $50 \%$ de los hospitales con maternidad tienen implementado el programa, dejando aproximadamente 47.000 niños a los cuales no se les realiza el examen de pesquisa auditiva universal. El progreso en establecer el TAU a nivel nacional como el estándar de cuidado debe ser un objetivo central de nuestra especialidad; y éste debe ser considerado como un programa integral, multidisciplinario, con seguimiento de los pacientes, más que como la simple realización de un examen.

6. US Preventive Services Task Force. Universal Screening for Hearing Loss in Newborns: US Preventive Services Task Force Recommendation Statement. Pediatrics Volume 122, Number 1, July 2008.

7. Joint Committe on Infant Hearing. 1994 Position Statement. Disponible en http://www.jcih.org/ JCIH1990.pdf.

8. Andrés Alvo V, Carolina Der M, Paul H. Délano R. Tamizaje universal de hipoacusia en el recién nacido. Rev Hosp Clín Univ Chile 2010; 21: 170-6.

9. Departamento de estadística e información DEIS Ministerio de Salud.

10. Krauss M, Karin et al. Programa de screening auditivo neonatal universal: Experiencia de más de 10 años. Rev. Otorrinolaringol. Cir. Cabeza Cuello [online]. 2013, vol.73, n.2, pp.125-132. ISSN 0718-4816. http://dx.doi.org/10.4067/ S0718-48162013000200003.

11. http://www.ine.cl/canales/menu/publicaciones/ calendario_de_publicaciones/pdf/completa_ vitales_2013.pdf [visita 5/11/2016].

12. IsaAcson, G. Candidate's Thesis: Universal Newborn Hearing Screening in an Inner-City, 
Managed Care Environment. The Laryngoscope 2000; 110: 881-94.

13. Kemper AR, Kus CA, Ostrander RJ, et al. A framework for key considerations regarding point-of-care screening of newborns. Genetics in medicine: Official Journal of the American College of Medical Genetics 2012; 14(12): 951954. doi:10.1038/gim.2012.89. 